\title{
REVISIÓN DE CONOCIMIENTOS SOBRE EL FRACASO RENAL AGUDO EN EL CONTEXTO DEL PACIENTE CRÍTICO
}

\author{
M. Romero-García ${ }^{1,2}$, P. Delgado-Hito ${ }^{2}$ L. de-la-Cueva-Ariza ${ }^{2}$ \\ ${ }^{1}$ Enfermería, Unidad de Cuidados Intensivos, Hospital de la Santa Creu i Sant Pau, Barcelona, España. \\ ${ }^{2}$ Escuela Universitaria de Enfermería, Universidad de Barcelona, España
}

\section{Resumen}

El fracaso renal agudo afecta entre un $1 \%$ y un $25 \%$ de los pacientes ingresados en unidades de cuidados intensivos, cifras que varían según la población y criterios estudiados.

Las complicaciones derivadas del fracaso renal agudo (hipervolemia, acidosis metabólica, hiperpotasemia, hemorragias) se tratan pero la mortalidad sigue siendo elevada a pesar de los avances tecnológicos de los últimos años ya que, habitualmente, el fracaso renal agudo está asociado a sepsis, insuficiencia respiratoria, heridas graves, complicaciones quirúrgicas o coagulopatías de consumo. El rango de mortalidad va desde un $30 \%$ a un $90 \%$.

Aunque no disponemos de una definición universalmente aceptada, la clasificación RIFLE aporta una herramienta operativa tanto para definir el grado de fracaso renal agudo como para homogeneizar el inicio de las Técnicas de Depuración Extrarrenal y evaluar los resultados obtenidos.

En consecuencia, las enfermeras que trabajan en una unidad de cuidados intensivos deben estar familiarizadas con esta patología, con su tratamiento (farmacológico o sustitutivo) y con la prevención de las posibles complicaciones. De igual manera han de ser capaces de detectar las manifestaciones de dependencia de cada una de las necesidades básicas e identificar los problemas de colaboración para conseguir un plan de cuidados individualizado.

Palabras clave: Cuidados intensivos, Atención de Enfermería, Proceso de Enfermería, Lesión Renal Aguda, Terapéutica.

\begin{abstract}
Acute renal failure affects between $1 \%$ to $25 \%$ of patients admitted to intensive care units, figures vary depending on the population studied and criteria.

Complications of Acute renal failure (fluid overload, metabolic acidosis, hyperkalaemia, bleeding) are treated but the mortality remains high despite technological advances in recent years because the acute renal failure is usually associated with sepsis, respiratory failure, serious injury, complications surgical or consumption coagulopathy. Mortality ranges from $30 \%$ to $90 \%$.

RIFLE classification gives us both an operational tool to define the degree of acute renal failure and to standardize the start of renal replacement techniques and evaluate the results.

We therefore consider that nurses working at intensive care unit should be familiar with this disease, with treatment (drug or alternative) and the prevention of possible complications. Must likewise be able to detect unmet needs depending on the manifestations of dependency, identify problems collaboratively to achieve individualized care plan.
\end{abstract}


Key words: Intensive Care, Nursing Care, Nursing Process, Acute Kidney Injury, Therapeutics. 


\section{INTRODUCCIÓN}

El Fracaso Renal Agudo (FRA) afecta entre un 1\% y un 25\% de los pacientes ingresados en Unidades de Cuidados Intensivos (UCI), cifras que varían según la población y criterios estudiados. No obstante, aunque pudiera parecer una cifra poco elevada es destacable el amplio rango de mortalidad (30\%$90 \%)^{(1,2,3)}$ que producen las complicaciones derivadas de este fracaso renal asociadas a la patología de base (sepsis, insuficiencia respiratoria, traumatismos graves, etc.).

En los últimos años, en las UCI, está aumentando un perfil de paciente con mayor probabilidad de presentar un FRA: con situaciones patológicas complejas, de edad más avanzada y con patología crónica de base. Además, la identificación, manejo y tratamiento de este trastorno se ha ido modificando a lo largo de estos últimos 10 años a través de reuniones de consenso de expertos, la primera realizada en agosto del 2000, que sugieren cambios en el manejo de este tipo de pacientes. Esta situación hace que el cuidado sea complejo y precise un mayor conocimiento de la fisiopatología, las manifestaciones clínicas y el tratamiento médico del FRA, con el fin de prevenir (en la medida de lo posible) y detectar precozmente las complicaciones potenciales que se puedan derivar mediante intervenciones y actividades enfermeras de mayor calidad y eficiencia.

En consecuencia, el objetivo de este artículo es actualizar los conocimientos sobre el FRA y las complicaciones potenciales relacionadas con el mismo en los pacientes ingresados en las UCI.

\section{FRACASO RENAL AGUDO: DEFINICIÓN Y ETIOLOGÍA}

El término de Insuficiencia Renal Aguda ha cambiado a FRA, para reflejar un continuum de la enfermedad y no como un solo evento ${ }^{(3,4,5)}$. Se define el FRA como un proceso progresivo y reversible, producido por causas hemodinámicas o tóxicas, que abarca desde el desarrollo de lesiones subletales de las células renales identificables por biomarcadores (fase de lesión renal aguda) al daño estructural caracterizado por la necroaptosis de las células tubulares, necrosis tubular aguda, en el que se constata elevación de la creatinina sérica y descenso de la tasa de filtrado glomerular ${ }^{(3)}$.

Esta disminución de la filtración lleva consigo la progresiva retención de agua y de productos nitrogenados (urea y creatinina) que habitualmente son eliminados por el riñón ${ }^{(6)}$.Se considera FRA oligúrico cuando el débito urinario es inferior a $5 \mathrm{ml} / \mathrm{Kg} / \mathrm{día}$ ( $400 \mathrm{ml} /$ día), no-oligúrico cuando es superior a $5 \mathrm{ml} / \mathrm{Kg} /$ día y anúrico cuando el débito urinario es menor a $0,6 \mathrm{ml} / \mathrm{Kg} /$ día $(50 \mathrm{ml} /$ día $)$.

La etiología de esta patología suele clasificarse en tres categorías: prerrenal (55\%-60\%), intrarrenal (35\%$40 \%)$ y posrenal $(<5 \%)$, siendo las causas prerrenales las más frecuentes en los pacientes ingresados en las $\mathrm{UCI}^{(7,8)}$. A continuación se describen las situaciones fisiopatológicas que se agrupan en cada una de estas categorías:

- FRA por causa prerrenal o funcional: Cualquier proceso que produzca una disminución de la presión arterial media renal y consecuentemente, la disminución de la presión de perfusión glomerular puede desencadenar un FRA por un defecto de la perfusión renal, como en el caso de pérdidas hemáticas, al producirse una disminución del volumen intravascular por hipovolemia. 
- FRA por causa intrarrenal o parenquimatosa: Los trastornos intrarrenales pueden ser debidos a la afectación de los glomérulos, de los túbulos, del intersticio o de los vasos intrarrenales. Una de las formas más característica y frecuente del FRA intrarrenal es la necrosis tubular aguda (NTA) o la nefropatía vasomotora representando alrededor del $70 \%-75 \%$ del total ${ }^{(9,10)}$. Existen dos mecanismos principales que originan NTA: la isquemia renal (FRA prerrenal prolongado) y la lesión tóxica renal directa por sustancias exógenas y endógenas.

- FRA por causa posrenal u obstructiva: El FRA de causa posrenal se produce debido a un aumento de la presión retrógrada haciendo disminuir el filtrado glomerular. La obstrucción puede producirse a cualquier nivel de las vías urinarias, desde el comienzo del sistema colector hasta el final de la uretra y puede estar originada por varias causas entre las que se encuentran litiasis, neoplasias o fibrosis retroperitoneales.

\section{FISIOPATOLOGIA}

Tal y como se ha mencionado con anterioridad la etiología prerrenal del FRA es la más frecuente en las personas ingresadas en la UCI ${ }^{(7,8)}$. Cuando se produce isquemia del riñón, el flujo renal queda seriamente comprometido, por lo que, la filtración disminuye $\mathrm{y}$, con esta reducción, aumentan los niveles de productos residuales del organismo manifestándose con un incremento de los niveles séricos de Nitrógeno Ureico en Sangre (BUN) y de creatinina. Otra de las consecuencias de la disminución del flujo renal es la reducción del aporte de oxígeno y de otros nutrientes vitales para el metabolismo celular.

En referencia a la tensión arterial sistólica, cuando se halla por debajo de $90 \mathrm{mmHg}$, el flujo sanguíneo renal, y como consecuencia el filtrado glomerular, presenta un descenso relacionado de forma directa con la cifra de la tensión. En el momento que los valores disminuyen por debajo de $50 \mathrm{mmHg}$, el filtrado glomerular cesa.

En los casos de hipoperfusión el riñón adopta dos medidas de adaptación que son la autorregulación y la liberación del sistema renina-angiotensina. La autorregulación consiste en la dilatación de la arteriola aferente (entrada de sangre al glomérulo) y la constricción de la arteriola eferente (salida de la sangre del glomérulo). Mediante este mecanismo se consigue aumentar el flujo sanguíneo en el interior del glomérulo y retrasar su salida, dando lugar a un aumento de la presión y de la velocidad de filtración glomerular ${ }^{\text {(Figura 1) }}$. Por otra parte, la liberación del sistema renina-angiotensina produce un aumento de la reabsorción del sodio y del agua lo que se traduce en un incremento del volumen intravascular y por consiguiente en una mayor perfusión renal ${ }^{(\mathrm{Figura} 2)}$.

Estos mecanismos o respuestas de adaptación son limitados y cuando los límites de los mismos se superan se instaura el FRA.

\section{GRADOS DEL FRA SEGÚN LA ESCALA RIFLE}

La escala Risk of renal dysfunction Injury to the kidney, Failure of kidney function, Loss of kidney function and End-stage kidney disease (RIFLE) ha sido consensuada por el grupo Acute Dialysis Quality Initiative $\mathrm{ADQI}$ en su $2^{\mathrm{a}}$ conferencia y aceptada a nivel europeo ${ }^{\text {(Figura } 3)}$. Esta clasificación aporta una herramienta operativa tanto para definir el grado de FRA como para homogeneizar el inicio de aplicación de las técnicas de depuración extrarrenal y evaluar los resultados obtenidos ${ }^{(10)}$. Posteriormente un nuevo grupo de trabajo, Acute Kidney Injury Network (AKIN), publicó otra definición y clasificación del FRA, existiendo controversia sobre cuál de las dos escalas predice mejor la mortalidad ${ }^{(6,10,11,12)}$. Se ha decidido presentar los grados del FRA según la escala RIFLE por ser la que se está utilizando con más frecuencia en los estudios publicados, especialmente a nivel europeo. 
Así, esta escala clasifica el FRA en tres grados de disfunción renal valorados por cifras bioquímicas o la Tasa de Filtrado Glomerular (TFG) y por el volumen de diuresis. Además añade dos niveles de pronóstico clínico: (i) el FRA persistente (Loss) que se define como la necesidad del uso de Técnicas de Depuración Extrarrenal más allá de las cuatro semanas y (ii) la enfermedad renal en estadio avanzado o terminal (Endstage) que se define por la necesidad de diálisis por encima de los tres meses. Esta clasificación hace hincapié en los siguientes conceptos: hay que considerar los cambios respecto al estado basal del paciente, se tiene que diferenciar entre enfermedad renal aguda o crónica, se ha de distinguir entre los casos leves o precoces (alta sensibilidad) y los graves o tardíos (alta especificidad), y su uso clínico ha de ser factible y reproducible entre diferentes centros ${ }^{(10,13)}$.

Los tres grados de disfunción renal definidos por la escala RIFLE, y descritos a continuación, son riesgo (Risk), lesión (Injury) y fallo (Failure).

\subsection{Riesgo}

Un alto porcentaje de casos de FRA podría evitarse si se tuviera en cuenta la edad del paciente, el estado hemodinámico y de hidratación, la función renal, el conocimiento sobre el manejo de fármacos nefrotóxicos y los pacientes en situación de riesgo: diabetes, mieloma múltiple o posoperatorio ${ }^{(6)}$. Para ello es necesario adoptar una serie de medidas generales y otras más específicas para cada caso en concreto (ver sección tratamiento preventivo). El tiempo de intervención es muy importante ya que sólo se puede actuar preventivamente durante esta primera fase del FRA ${ }^{(8,14,15)}$.

\subsection{Lesión}

Esta fase comprende desde la lesión o agresión inicial hasta la aparición de la uremia aguda (fallo). Puede durar entre unas horas o algunos días y tiene una importancia significativa ya que una actuación inmediata es capaz de resolver, o prevenir, la evolución al siguiente estadio, el fallo renal. Por tanto un diagnóstico y tratamiento precoz seria lo idóneo dentro de esta fase. No obstante, el curso clínico del FRA dependerá en gran parte de la etiología subyacente.

\subsection{Fallo}

La aparición de la uremia debido a la disminución de la función excretora normal sería lo más destacable en este grado. Esta fase suele durar entre 8-20 días. El débito urinario es no-oligúrico en el 30\%-50\% de los paciente, oligúrico en la mayoría restante y aparece anuria entre el 3\%-10\% de los pacientes ${ }^{(8)}$. Los pacientes que presentan oliguria o anuria tienen una evolución clínica más prolongada y complicaciones más frecuentes y graves que los pacientes en situación de no-oliguria. Asimismo, en la fase oligúrica de la FRA es donde se registra la mayor tasa de mortalidad $^{(9)}$.

A los 14 días después de la lesión inicial, se inicia una fase diurética que suele durar aproximadamente unos 10 días y señala la recuperación de las nefronas y de su capacidad para excretar orina (gasto urinario $>1000 \mathrm{ml} /$ día). Por lo general, la diuresis se inicia antes de que las nefronas se hayan recuperado por completo por lo que se sigue manteniendo la azoemia (niveles séricos elevados de BUN y creatinina) llegando a necesitar algunas sesiones de diálisis.

Esta recuperación suele durar entre 6 y 12 meses. La reparación y la regeneración del tejido renal, está precedido clínicamente por un incremento gradual de la diuresis que, generalmente, es ineficaz en los primeros días. Al cabo de cierto tiempo, la diuresis se hace eficaz y comienza el descenso de las cifras de urea y creatinina, y la recuperación del filtrado glomerular. Este incremento en la diuresis puede producirse de forma paulatina o bruscamente, de un día para otro, pudiendo producir poliuria superior a 45 l/día y por consiguiente alteraciones hidroelectrolíticas importantes. A pesar de ello, cabe destacar que, aproximadamente un 5\% de los que han presentado necrosis tubular, no llegan a recuperar totalmente la función renal ${ }^{(9)}$. 


\section{MANIFESTACIONES CLÍNICAS DEL FRA}

Es en el grado de disfunción correspondiente al fallo renal, cuando aparece el síndrome urémico, o conjunto de signos y síntomas, producido por el acúmulo de productos residuales relacionados con la incapacidad del riñón para mantener sus funciones. Una de las manifestaciones bioquímicas de esta fase es el aumento del BUN (de 10 a $20 \mathrm{mg} / \mathrm{dl} /$ día) y de la creatinina (de 0 '5 a $1 \mathrm{mg} / \mathrm{dl} /$ día), ambos productos residuales del metabolismo. En casos de sepsis o traumatismos, la tasa de incremento del BUN puede llegar a exceder los 100mg/dl/día. En los traumatismos, y como consecuencia de una destrucción muscular, el aumento de la creatinina también puede ser superior.

A medida que progresa el cuadro urémico el estado de conciencia se verá afectado. Inicialmente se aprecia un cierto torpor mental que dificulta la comunicación. Posteriormente aparecen, de forma progresiva: letargia, confusión, estupor, agitación, psicosis, mioclonias, hiperreflexia, síndrome de piernas inquietas, déficit neurológico focal, convulsiones y por último, el coma ${ }^{(8)}$.

Asimismo, la toxicidad urémica puede ocasionar manifestaciones gastrointestinales (hipo, náuseas, vómitos, anorexia) y alteraciones que, inicialmente, no tienen mucha repercusión como son las relacionadas con el sistema endocrino y reproductivo.

Aparece también en este periodo la acidosis metabólica como resultado de la liberación de grandes cantidades de hidrogeniones (principalmente en forma de ácido sulfúrico y fosfórico) y de la disminución de la producción de bicarbonato en las células renales. En esta situación los pulmones intentarán compensar el pH hiperventilando, eliminando el exceso de anhídrido carbónico (respiración de Kussmaul) pudiendo aparecer síntomas neurológicos como la somnolencia que progresa a estupor y coma.

Siguiendo con las complicaciones propias de este estadio del FRA, la hiperpotasemia o hipercaliemia es considerada una de las más graves. Se produce por la salida de potasio intracelular al líquido extracelular inducida por las lesiones hísticas, el catabolismo y la acidosis. Además, el cuadro puede agravarse por cargas de potasio exógenas en forma de antibióticos que contengan potasio, sustitutos de la sal y varios fármacos que afectan la distribución extrarrenal del potasio como los betabloqueantes, los inhibidores de la enzima convertidora, la heparina y/o los diuréticos ahorradores de potasio ${ }^{(8,9)}$. Los mejores indicadores de la hiperpotasemia son los cambios en el ECG y la determinación de los niveles séricos mayores de 5'5 $\mathrm{mEq} / \mathrm{l}^{(16)}$.

La hipocalcemia y la hiponatremia son también manifestaciones del FRA. La hiponatremia se produce como resultado del exceso de líquidos (ya sean por vía oral o endovenosa), de la destrucción hística (liberación importante de agua al espacio extracelular) y de la liberación de agua por el catabolismo endógeno de grasas y proteínas (300-500 ml/día). La hipocalcemia se produce por un aumento en sangre de fósforo producido a causa de la dieta y la liberación de fósforo de los tejidos, incrementada por las lesiones hísticas y la acidosis.

La hipertensión arterial, el edema y la insuficiencia cardíaca congestiva aparecen frecuentemente en el FRA complicado. Aparecen como resultado de la retención de agua y sal a causa de las alteraciones hidro-electrolíticas. Habitualmente se equipara el aumento de $1 \mathrm{Kg}$. de peso con una retención de 1 litro de agua. En la actualidad, y debido en gran parte a la instauración de la diálisis, otras alteraciones como la pericarditis o el taponamiento cardíaco son infrecuentes.

En cuanto a las manifestaciones hematológicas que puede presentar un paciente con FRA destacaremos la anemia (secundaria a la disminución de eritropoyetina, la hemólisis, la corta vida de los eritrocitos y las pérdidas sanguíneas), la diátesis hemorrágica (como resultado de las alteraciones en la agregación y adhesividad plaquetaria) y las alteraciones del sistema inmunológico (secundario a los efectos tóxicos de la urea sobre el sistema inmunitario) lo que favorece la aparición de infecciones. 
En la tabla 1, se presenta una clasificación de las manifestaciones clínicas del fallo renal en base a algunas de las necesidades básicas de la persona según el modelo de Henderson ${ }^{(17)}$, que más frecuentemente se encuentran alteradas sobre todo en su dimensión física. Por otro lado, es preciso mencionar que, en esta tabla no se han incluido las manifestaciones de dependencia e independencia dado que el artículo no refleja un proceso de cuidados enfermeros individualizado a una persona en concreto como otros autores ya han publicado $^{(18)}$.

\section{TRATAMIENTO DEL FRA}

El tratamiento del FRA está dirigido, en primer lugar, a la prevención utilizando medidas que permitan reducir el riesgo de lesión renal en aquellas situaciones candidatas a desarrollar un FRA (cirugía mayor con cambios hemodinámicos, shock séptico, hemorrágico o cardiogénico, rabdomiolisis, contrastes radiológicos o nefrotóxicos, lisis tumoral, etc.); en segundo lugar, al tratamiento de las causas que puedan hacer progresar una lesión renal; en tercer lugar, al tratamiento de las consecuencias de un FRA ya establecido; y en cuarto lugar, al tratamiento sustitutivo mediante técnicas dialíticas.

\subsection{Tratamiento profiláctico o preventivo}

Existen diferentes situaciones en las que se puede actuar para disminuir el riesgo de generar una lesión renal posterior en las personas ingresadas en unidades de cuidados intensivos. La primera medida preventiva es la identificación de aquellos pacientes que presentan factores de riesgo como la edad avanzada, la diabetes, la hepatotía crónica o la cardiopatía descompensada ${ }^{(3,11)}$.

Así, cuando un paciente está en shock será necesario reponer volumen (mediante la infusión de cristaloides, coloides o hemoderivados según la etiología) y, si con una expansión de volumen adecuado no mejora, será necesario la utilización de drogas inotrópicas que mejoran el gasto cardíaco y por tanto la perfusión renal. La utilización de noradrenalina en perfusión continua sigue siendo el fármaco vasopresor de elección. Por otro lado, es preciso tener en cuenta que la administración de dopamina a dosis bajas se considera, en la actualidad, una contraindicación en el tratamiento del FRA ${ }^{(3)}$.

Por otro lado, en la medida de lo posible, hay que evitar el uso de medicamentos nefrotóxicos. Cuando un paciente necesita ser tratado con fármacos nefrotóxicos, como es el caso de la amikacina o la gentamicina, será necesario monitorizar cuidadosamente los niveles séricos de estos medicamentos y adaptar las dosis según los resultados obtenidos (la eliminación del fármaco puede variar en los pacientes críticos) y a la vez, realizar controles analíticos de creatinina y urea ${ }^{(7,8,19,20)}$. Además, la administración de amino glucósidos en una sola dosis al día produce menor nefrotoxicidad que el tratamiento convencional con tres dosis al día y es igualmente eficaz ${ }^{(21,22)}$. En conclusión, es preciso ajustar las dosis, o intervalos de dosificación, a la función renal del paciente, controlando sus niveles en sangre y manteniendo una correcta hidratación evitando, siempre que sea posible, el uso de más de un fármaco nefrotóxico.

Cuando se requieran medios de contraste para realizar pruebas radiológicas concretas se realizará una correcta hidratación del paciente antes de la exploración ${ }^{(3,19,23)}$. La administración de una carga de volumen de suero fisiológico al $0,45 \%$ en los pacientes de riesgo antes de que sean expuestos a contrastes radiológicos debería ser una orden médica inicial ${ }^{(19,20,24)}$. Además, se ha de utilizar la menor cantidad de contraste posible y adaptarlo a la función renal. Al cabo de 48 horas se realizará un análisis en sangre para controlar los valores de creatinina.

En el caso de que presenten una rabdomiolisis, especialmente de origen traumático, el hecho de forzar la diuresis (administrando volumen) y de alcalinizarla (administrando bicarbonato sódico endovenoso) también disminuye el riego de sufrir fracaso renal $^{(3,19)}$. 
Es evidente que disminuir la mortalidad y mejorar el pronóstico funcional de la función renal requiere de un tenaz esfuerzo encaminado a la prevención, el diagnóstico y el tratamiento precoz de las situaciones de riesgo, como la hipotensión, la nefrotoxicidad, la sepsis o algunos tipos de cirugías ${ }^{(3)}$, por parte de todos los profesionales implicados en la cadena asistencial.

\subsection{Tratamiento etiológico}

Es preciso valorar que el volumen intravascular sea el adecuado ( $\mathrm{PVC}^{1} 10-12 \mathrm{mmHg}$ ó $\mathrm{PCP}^{2} 15-20 \mathrm{mmHg}$ ). Cuando aparezca oliguria y valores de PVC ó PCP inferiores a los descritos, se repondrá volumen hasta conseguir valores normales, si aún así persiste la oliguria se utilizarán drogas inotrópicas en un intento de mejorar el gasto cardíaco ${ }^{(4,7,8,23)}$. Es importante descartar, previamente, que la alteración de la diuresis no se deba a la presencia de globo vesical o de obstrucción del sondaje vesical ${ }^{(4)}$.

La administración de furosemida en una perfusión endovenosa continua es mejor que la administración en bolus para evitar la ototoxicidad ${ }^{(25)}$ además de mejorar la respuesta diurética y de producir menos efectos adversos ${ }^{(26)}$. En contrapartida, la utilización de manitol o furosemida como diuréticos no parece proporcionar ningún beneficio adicional ${ }^{(3,4,7,27)}$. Según la ADQI, el tratamiento ideal es una correcta hidratación sin diuréticos.

\section{COMPLICACIONES POTENCIALES MÁS FRECUENTES EN EL PACIENTE CON FRA Y CUIDADOS ENFERMEROS}

\subsection{Complicaciones potenciales referentes al FRA}

Según Luis ${ }^{(28)}$, los Problemas de Colaboración se refieren a las Complicaciones Potenciales (CP) de la situación fisiopatológica, del tratamiento prescrito o de las pruebas diagnósticas hechas. Los objetivos de la enfermera se referirán siempre a la instauración de medidas encaminadas a la prevención de las complicaciones y la detección temprana de signos y síntomas indicadores de su aparición. Las CP que con mayor frecuencia presentan los pacientes con FRA ingresados en las UCI y las actividades enfermeras ${ }^{(29)}$ asociadas a las mismas son las siguientes:

\subsubsection{CP: Desequilibrio hidroelectrolítico secundario a Fallo Renal Agudo.}

Los trastornos hidroelectrolíticos son todas aquellas alteraciones del contenido corporal de agua o electrolitos en el cuerpo humano. Se clasifican según sean por defecto o por exceso y también contempla las alteraciones del equilibrio ácido-base. Las actividades enfermeras se centrarán en:

- Registrar las entradas y salidas de líquidos realizando un balance hídrico estricto diario.

- Control diario del peso del paciente. El peso es un indicativo más de la ganancia o pérdida de líquidos.

- Reponer líquidos de forma adecuada evitando la hiperhidratación.

- La reposición de líquidos será equivalente a las pérdidas insensibles más las pérdidas urinarias más las pérdidas por otras vías. Es importante adecuar el balance hídrico y electrolítico $^{(30)}$.

- Normalmente una persona ingresada en UCI es portadora de múltiples vías con perfusiones continuas de sueroterapia y de medicaciones necesarias para el tratamiento de su patología. En este contexto es preciso realizar una restricción hídrica estricta para evitar una sobrecarga de volumen, por tanto, las diluciones de las medicaciones administradas se realizaran siempre con el mínimo volumen posible.

\footnotetext{
${ }^{1}$ Presión Venosa Central

${ }^{2}$ Presión Capilar Pulmonar
} 
- Detectar signos y síntomas de sobrecarga hídrica: edema en zonas de declive, ingurgitación yugular, ganancia de peso.

- Detectar signos y síntomas de edema agudo de pulmón ${ }^{1}$ : disnea, taquicardia, ruidos respiratorios (crepitantes) secreciones rosadas y espumosas, aumento de las presiones endocavitarias, cianosis y aumento de la tensión arterial.

- Valorar niveles séricos y en orina de electrolitos.

- Detectar signos y síntomas de la hiperpotasemia:

- Trastornos cardíacos: monitorización y control del electrocardiograma. La hiperpotasemia es la complicación electrolítica más grave de la FRA. La alteración del potasio tiene una repercusión directa sobre la conducción eléctrica cardiaca pudiendo producir trastornos del ritmo, onda $\mathrm{T}$ picuda, disminución de la amplitud de la onda $\mathrm{P}$, depresión del segmento ST y un ensanchamiento del complejo QRS. En las situaciones más graves se observa asistolia atrial y bloqueos intraventriculares que pueden derivar en una parada cardiaca.

- Trastornos neurológicos: irritabilidad, ansiedad, debilidad muscular, hiporreflexia, parálisis flácida, entumecimiento y hormigueos.

- Trastornos gastrointestinales: náuseas, vómitos, retortijones abdominales.

- Administración del tratamiento médico prescrito para disminuir los niveles séricos de potasio.

- El tratamiento medicamentoso para la hiperpotasemia puede incluir la administración de los siguientes fármacos: (1) cloruro cálcico endovenoso (e.v.) como antagonista del efecto tóxico del potasio sobre la conducción cardiaca con una efectividad rápida; (2) glucosa e insulina e.v. cuyo efecto combinado facilita el paso de potasio al espacio intracelular, siendo esta medida efectiva durante varias horas; (3) salbutamol e.v. cuya administración requiere el control exhaustivo del electrocardiograma durante la misma debido a su principal efecto secundario, el aumento de la frecuencia cardíaca; (4) de bicarbonato sódico e.v. que actúa proporcionando sodio como antagonista de los efectos del potasio sobre el corazón y deposita el potasio en el espacio intracelular al corregir la acidosis; (5) la administración de resinas de intercambio catiónico por vía oral o vía rectal mediante enemas, facilitando la excreción de potasio a la luz intestinal ${ }^{(8,20)}$. En este último caso, para asegurar la efectividad del enema se dejará durante al menos 1-2 horas y previa siguiente aplicación se realizará un enema de limpieza.

- Detectar signos y síntomas de otras alteraciones iónicas asociadas a alteraciones del calcio, fósforo y sodio.

- Manifestaciones de hipocalcemia: irritabilidad, tetania muscular, signo de Chvostek (espasmo muscular facial), signo de Trousseau (espasmo carpal), entumecieminto y hormigueo periférico, calambres musculares, convulsiones, alucinaciones, confusión, disminución del gasto cardíaco, prolongación del intervalo QT y cambios en la onda $\mathrm{T}^{(8)}$, hemorragias.

- Manifestaciones de hiperfosfatemia: Sensación de hormigueo, anorexia, náuseas, vómitos, debilidad muscular.

- Manifestaciones de hiponatremia: neurológicas o neuromusculares como confusión, delirio, letargia, coma, jaquecas, temblores, convulsiones, debilidad muscular, hiperreflexia entre otros. Puede aparecer un enrojecimiento cutáneo, piel caliente y húmeda.

- Administración del tratamiento médico prescrito para corregir los niveles séricos de otros iones:

- Tratamiento de la hipocalcemia: La hipocalcemia no necesita tratamiento excepto si es sintomática. Los fármacos utilizados para aumentar los niveles de calcio serán el

\footnotetext{
${ }^{1}$ El edema agudo de pulmón puede tratarse como una CP en sí misma: CP: Edema agudo de pulmón secundario a sobrecarga hídrica.
} 
Carbonato Cálcio, Cloruro Cálcico y Gluconato Cálcico vía endovenosa diluidos según prescripción y administrados lentamente.

- Tratamiento de la hiperfosfatemia con quelantes de fósforo por vía oral como el hidróxido de aluminio (que disminuyen la absorción intestinal de fosfato) ${ }^{(8)}$. Se tendrá en cuenta el efecto astringente de estos fármacos y por lo tanto la prevención del estreñimiento.

- Tratamiento dietético para la conservación del equilibrio iónico:

- Control de la hiperfosfatemia con restricción de alimentos ricos en fósforo.

- Control de la hiponatremia con dieta hiposódica y restricción de líquidos ya que suele ser consecuencia de la administración de sueros glucosados hipotónicos ${ }^{(7)}$.

- Realizar tratamiento sustitutivo renal según prescripción médica siguiendo el protocolo establecido.

- Administración del tratamiento médico prescrito para corregir acidosis metabólica. La acidosis metabólica aparece cuando el riñón es incapaz de eliminar los ácidos generados en situaciones hipercatabólicas propias de sepsis, politraumatismos o grandes quemados. Cuando los esfuerzos respiratorios para mejorar la acidosis mediante la hiperventilación son insuficientes el tratamiento se basa en la administración de bicarbonato sódico o la diálisis (necesaria cuando la producción de ácidos es muy elevada o cuando la sobrecarga de volumen impide dar la cantidad de bicarbonato requerida).

- Se trata sólo cuando los niveles plasmáticos de bicarbonato son inferiores a $15 \mathrm{mEq} / \mathrm{l}^{(8,23)} \mathrm{o}$ cuando el Ph sanguíneo es inferior a $7,2^{(7)}$.

7.1.2 CP: Infección secundaria a alteración del sistema inmunitario y/o procedimientos invasivos.

La infección es un fenómeno microbiano caracterizado por una respuesta inflamatoria a la presencia de microorganismos o la invasión de tejidos estériles del huésped por dichos microorganismos. Es la complicación más importante del FRA, se presentan en el 30-70\% de los casos y es la causante del 75\% de las muertes ${ }^{(7)}$. La enfermera tendrá en cuenta lo siguiente:

- Aplicación de medidas asépticas. Las infecciones se ven favorecidas por roturas de las barreras mucocutáneas (catéter intravenosos, intubación traqueal, sondas urinarias) y por alteraciones de la inmunidad asociadas a la uremia.

- El tratamiento sería la prevención mediante una asepsia estricta en los cuidados realizados y la protección de otros pacientes que sufren infecciones mediante un aislamiento preventivo.

- Es fundamental evitar en lo posible la existencia de focos de entrada, retirar el tratamiento invasivo lo antes posible ${ }^{(8)}$.

- Identificar signos y síntomas de infección:

- Control regular de la temperatura.

- Identificación de manifestaciones de infección localizada: En punto de inserción de vías invasivas y heridas (enrojecimiento, tumefacción, calor local y supuración), en vías respiratorias (tos, estornudos, secreción purulenta, alteraciones del patrón respiratorio), en vía urinaria (disuria, orina turbia, piuria, etc.)

- Identificación de manifestaciones de infección sistémica: fiebre, temblor generalizado acompañado de hipertensión arterial y subida súbita de la temperatura.

- Administración de antibióticos según pauta médica:

- No se recomienda el uso profiláctico de antibióticos ${ }^{(8)}$. Si la persona presenta una infección se administra antibiótico según el agente causal, teniendo en cuenta que el riñón es la vía de excreción de numerosos fármacos ${ }^{(8,31)}$.

- La administración de estos fármacos se realizará dentro de los 15 minutos de la hora programada para mantener los niveles en sangre adecuados. 
- Extracción de cultivos si procede.

7.1.3. CP: Hemorragia secundaria a alteraciones en la agregación y adhesividad plaquetaria y/o tratamiento anticoagulante.

Los cuidados enfermeros incluyen:

- Minimizar el riesgo de producción de heridas (afeitar con maquinilla eléctrica, utilizar cepillo de dientes suave, etc).

- Realizar los mínimos procedimientos invasivos que requiera el estado del paciente.

- Detección precoz de signos y síntomas de hemorragia:

- Presencia de hematomas, epistaxis, sangrado de encías, hematuria, hemoptisis, sangrado gástrico, cefalea intensa o alteraciones neurológicas, heces melénicas, palidez de piel y mucosas, aumento del débito hemático en drenajes.

- Control hemodinámico para la identificación de taquicardia, hipotensión, disminución de las presiones endocavitarias.

- Detección de manifestaciones propias de shock hemorrágico: hipotensión arterial severa, pulso rápido y filiforme, taquipnea, diaforesis, inquietud, piel fría y sudorosa, sensación de muerte inminente.

- Control analítico, según frecuencia precisada, de niveles de hemoglobina, hematocrito y pruebas de coagulación.

- Administración del tratamiento médico prescrito para la prevención de hemorragias:

- Fármacos de protección gástrica. El riesgo de hemorragia digestiva debido a los efectos tóxicos de la urea sobre las plaquetas se previene con medidas de protección gástrica (alcalinos), aún así aparece en un 10-30\% de los $\operatorname{casos}^{(31)}$.

- Administración de protamina, según pauta, en caso de hemorragia importante causada por la administración de heparina.

7.1.4. CP: Desnutrición secundaria a las náuseas, vómitos, inapetencia o restricciones dietéticas.

Entre los cuidados enfermeros destacan:

- Detección precoz de signos y síntomas de desnutrición:

La desnutrición se inicia cuando los pacientes se someten a una ingesta inadecuada, no sólo por las dificultades en el aporte de la misma al concurrir en ellos muchos factores limitantes como la caquexia, anorexia, náuseas, vómitos, sino también por las frecuentes restricciones en el aporte proteico a las que se somete a los enfermos críticos ${ }^{(32)}$.

- Determinación diaria de peso.

- Determinar junto a la dietista el número de calorías y el tipo de nutrientes necesarios para satisfacer las exigencias de alimentación.

- El objetivo nutricional de los pacientes con FRA supone un adecuado aporte calórico (30$50 \mathrm{Kcal} . / \mathrm{Kg} . / \mathrm{día}$ ) para evitar el catabolismo y la cetoacidosis ${ }^{(7)}$, reduciéndose a su vez la producción de residuos nitrogenados, ya que la urea es un producto de degradación de las proteínas y, proporcionando la mayor parte de las calorías en forma de carbohidratos (100g/día)..

- Algunos pacientes pueden necesitar una ingesta proteica superior, en especial si están hipercatabólicos o el FRA tiene un curso prolongado. En este caso el médico valorará la necesidad de aumentar dicha ingesta aunque esto precipite la necesidad de diálisis, con el fin de evitar que se produzca una desnutrición ${ }^{(8,30,31)}$.

- Las vías de administración pueden ser enteral o parenteral, siendo la vía enteral la de primera opción.

- Valoración de la tolerancia de la dieta enteral.

- Administración de dieta parenteral, según protocolo establecido si se precisara. No está demostrado que una hiperalimentación parenteral varíe el pronóstico del FRA $^{(8,30)}$ pero sí en 
cambio que la desnutrición comporta un incremento de la morbimortalidad ${ }^{(7)}$ y un deterioro de la inmunidad ${ }^{(32)}$.

- Administración de antieméticos, según pauta, en caso de existir náuseas y/o vómitos.

\section{CONCLUSIÓN}

Este artículo ha pretendido ser un medio para actualizar los conocimientos sobre el FRA y las complicaciones potenciales asociadas al mismo. En este sentido se ha presentado una actualización de conocimientos relativos a la definición, etiología, fisiopatología, grados de disfunción renal según la escala RIFLE, manifestaciones clínicas y tratamiento. Es preciso mencionar que la definición de los grados del FRA se ha realizado en base a la escala RIFLE por ser ésta una herramienta operativa utilizada para homogeneizar el inicio de las técnicas de depuración extrarrenal y evaluar los resultados obtenidos.

Por otro lado, el desarrollo de las complicaciones potenciales más frecuentes en pacientes con FRA con actividades enfermeras dirigidas a la prevención (cuando sea posible) y a la detección precoz de las mismas puede ser una guía para dispensar cuidados adecuados a un paciente crítico cada vez más complejo. En este sentido, un alto grado de preparación teórica permitirá la instauración de medidas de control y vigilancia del estado del paciente, tanto en lo que respecta a la evolución de su situación clínica como a los efectos de los tratamientos prescritos, dando lugar a unos cuidados enfermeros de gran calidad. 


\section{BIBLIOGRAFÍA}

1. Kellum JA, Levin N. Bouman C, et al. Developing a consensus classification system for acute renal failure. Curr Opin Crit Care. 2002; 8:509-514.

2. Mehta RL, Kellum JA, Shah SV, et al. Acute Kidney Injury Network: report of an initiative to improve outcomes in acute kidney injury. Crit Care. 2007;11(2):R31.

3. Andrés E. Prevención primaria y secundaria del fracaso renal agudo. En Net À, Roglán A, editores. Disfunción Renal Aguda en el paciente Crítico. Barcelona: Ars Medica;2009.p. 31-53.

4. Rhonda K. Acute Kidney Injury. Advances in definition, pathophysiology, and diagnosis. AACN Adv Crit Care. 2010;21(4):350-356

5. Dirkes S. Acute kidney Injury: not just acute renal failure anymore? Crit Care Nurs 2011;31(1):37-50.

6. Abuelo JG. Normotensive ischemic acute renal failure. N Engl J Med: 2007; 35 (7): 797-805

7. Morán I, Roglán A, Barcons M. Insuficiencia renal aguda. Depuración renal. En Lloret J, Muñóz J, Artigas V, Allende LH, Vázquez G, editores. Protocolos terapéuticos de urgencias. $5^{\mathrm{a}}$ ed. Barcelona: Masson; 2004.p.271-277.

8. Rivero M, Rubio J, Cózar J, García D. Insuficiencia renal aguda. En Gil J, Díaz-Alersi R, Coma MJ, Gil D, editores. Principios de Urgencias, Emergencias y Cuidados Críticos. 2002. [Acceso 31 de marzo 2010] Disponible en:

http://tratado.uninet.edu/indautor.html

9. Rozman C. Farreras. Medicina Interna. 15 ed. Barcelona: Elsevier;2004

10. Liaño F, Tenorio MT, Rodríguez N. Clasificación, epidemiología y diagnóstico de la insuficiencia renal aguda. En Net À, Roglán A, editores. Disfunción Renal Aguda en el paciente Crítico. Barcelona: Ars Medica; 2009. p.1-14.

11. Alcázar R, de la Torre M, Santana H. Avances en la insuficiencia renal aguda en el año 2008. Nefrología. 2009; 29(sup.Ext.5):82-87.

12. Cruz J, Cruz C, Sánchez M, Estrada C. Insuficiencia renal aguda en la Unidad de Cuidados Intensivos. Revisión. Revista de la Asociación Mexicana de Medicina Crítica y Terapia Intensiva. 2009; 13(2):89-93

13. Bellomo R, Ronco C, Kellum JA, Mehta RL, Palevsky P. Acute renal failure-definition, outcome measures, animal models, fluid therapy and information technology needs: the Second International Consensus of the Acute Dialysis Quality Initiative (ADQI) Group. Crit Care. 2004;8(4): R204-R212

14. Herrera ME, Seller G, Maynar J, Sanchez-Izquierdo JA. Epidemiology of acute Kidney failure in Spanish ICU. Multicenter prospective study FRAMI. Med Intensiva 2006;30:260-267.

15. Liaño F, Álvarez L, Rodríguez J. Epidemiología de la insuficiencia renal aguda. Nefrología. 2007;27(3):6-14.

16. Beare PG, Miers JL. Principios y Práctica de la enfermería médico-quirúrgica. $2^{\mathrm{a}}$ ed. (vol. 1, cap4). Madrid: Mosby-Doyma;1995.p.995-1060

17. Luis MT, Fernández C, Navarro MV. De la teoría a la práctica: El pensamiento de Virginia Henderson en el siglo XXI. $3^{a} E d$. Barcelona: Masson;2005.

18. Delgado P, Romero M. Plan de Cuidados en un Paciente con Insuficiencia Renal Aguda. Enfermería Nefrológica 1999; 5: 46-52.

19. Gainza F.J. Liaño F. Actuación en el Fracaso Renal Agudo. Guías SEN. Nefrología. 2007, 27. Suplemento 3.

20. Van Biesen W, Lamiere N. Nuevos conocimientos relativos a la fisiopatología y la prevención de la necrosis tubular aguda en la unidad de cuidados intensivos. En Net À, Roglán A, editores. Depuración extrarrenal del paciente grave. Barcelona: Masson; 2004.p.89-98.

21. Gilbert DW. Once-daily aminoglycoside therapy. Antimicrobial Agents. Chemother, 1991; 35: 399-405. 
22. Prins JM, Buller HR, Speelman P, Kuijper EJ, Tange RA. Once versus thrice daily gentamicin in patients with serious infections. Lancet.1993;341: 335-339.

23. Alía, I. Insuficiencia renal aguda. En Esteban A, Martín C, editores. Manual de cuidados intensivos para enfermería. $3^{\text {a }}$ ed. Barcelona: Springer-Verlag Ibérica;1996.p.217-226

24. Baker CSR, Wragg A, Kumar S, De Palma R, Bake, LRI, Knight CJ. A rapid protocol for the prevention of contrast-induced renal dysfunction: The RAPPID Study. J Am Coll Cardiol, 2003; 41: 2114-2118.

25. Barcons M, Roglán A. Fracaso renal agudo. En Lloret J, Muñoz J, Artigas V, Allende LH, Anguera I. Protocolos terapéuticos de Urgencias, Hospital de la Santa Creu i Sant Pau. 3 ed. Barcelona: Springer;1997.p.375-381.

26. Martin SJ, Danziger LH. Continuous infusion of loop diuretics in the critically ill: a review of the literature. Crit Care Med 1994;22:1323-1329.

27. Mehta RL, Pascual MT, Soroko S, Chertow GM. Diuretics, mortality, and nonrecovery of renal function in acute renal failure. JAMA 2002;288(20): 2547-2553.

28. Luis MT. Los diagnósticos enfermeros: revisión crítica y guía práctica. $8^{\mathrm{a} e d . ~ B a r c e l o n a: ~ M a s s o n ; ~}$ 2008.

29. Bulechek G, Butcher $\mathrm{H}$, McCloskey J. Clasificación de intervenciones de enfermería. $5^{\mathrm{a}}$ ed. Barcelona: Elsevier; 2009.

30. Torras, A. Fisiopatología y diagnóstico del fracaso renal agudo. En Net À, Roglán A, editores. Fracaso renal agudo. Barcelona: Springer-Velarg Ibérica;1999.p.1-19.

31. Montoliu, J. Prevención y tratamiento del fracaso renal agudo. En Net À, Roglán A, editores. Fracaso renal agudo. Barcelona: Springer-Velarg Ibérica;1999.p.59-65.

32. López J, Heras A. Nutrición del enfermo crítico. Soporte nutricional especializado en la insuficiencia renal. Cap. 18. En: Libro electrónico de Medicina Intensiva. Ed. 1 ${ }^{\text {a }}$. 2008. [acceso 18 de abrilde2011]. Disponible en:

http://www.medicina-intensiva-libro.com/2011/03/618-soporte-nutricional-especializado.html 
Tablas:

Tabla 1. Realizar cuidados enfermeros a partir de las manifestaciones clínicas

Necesidad de respirar

\begin{tabular}{|c|c|}
\hline Manifestaciones clínicas & Mecanismo fisiopatológico \\
\hline $\begin{array}{l}\text { - Alteraciones } \\
\text { electrolíticas }\end{array}$ & - Excreción disminuida \\
\hline - Acidosis metabólica & $\begin{array}{l}\text { Secreción hidrogeniones disminuida, } \\
\text { reabsorción y generación de bicarbonato } \\
\text { disminuida, excreción de sales de fosfato } \\
\text { disminuida }\end{array}$ \\
\hline - Hipertensión & $\begin{array}{l}\text { Exceso de líquidos, retención de sodio } \\
\text { incrementada, inapropiada activación del } \\
\text { sistema renina-angiotensina }\end{array}$ \\
\hline $\begin{array}{l}\text { - Insuficiencia cardíaca } \\
\text { congestiva }\end{array}$ & - Exceso de líquidos, hipertensión \\
\hline - Arritmias & $\begin{array}{l}\text { - Alteraciones electrolíticas (potasio, calcio) y } \\
\text { variaciones en el sodio }\end{array}$ \\
\hline - Edema pulmonar & $\begin{array}{l}\text { - Exceso de líquidos } \\
\text { - Permeabilidad de los capilares pulmonares } \\
\text { - } \text { aumentada } \\
\text { - Disfunción del ventrículo izquierdo }\end{array}$ \\
\hline - Neumonía & $\begin{array}{l}\text { - Secreciones orales espesas secundario al } \\
\text { - Paciente aletargado con una disminución del } \\
\text { reflejo tusígeno } \\
\text { - Actividad macrófaga pulmonar disminuida } \\
\text { - Exceso de líquidos }\end{array}$ \\
\hline $\begin{array}{l}\text { - Respiración de } \\
\text { Kussmaul }\end{array}$ & $\begin{array}{l}\text { - Aumento de la frecuencia respiratoria para } \\
\text { compensar la acidosis metabólica }\end{array}$ \\
\hline
\end{tabular}


Necesidad de eliminar

\begin{tabular}{|l|l|}
\hline Manifestaciones clínicas & \multicolumn{1}{c|}{ Mecanismo fisiopatológico } \\
\hline - Exceso de líquidos & - Excreción disminuida \\
\hline - Problemas intestinales: & - Efectos tóxicos de la urea \\
& $\begin{array}{l}\text { Hiarrea o constipación } \\
\text { electrolíticas especialmente la hipercaliemia } \\
\text { Hipomotilidad secundaria a las alteraciones } \\
\text { electrolíticas, disminución de la ingesta } \\
\text { hídrica y la actividad }\end{array}$ \\
\hline
\end{tabular}

Necesidad de nutrición e hidratación

\begin{tabular}{|l|l|}
\hline Manifestaciones clínicas & \multicolumn{1}{c|}{ Mecanismo fisiopatológico } \\
\hline $\begin{array}{l}\text { Anorexia, náuseas y } \\
\text { vómitos }\end{array}$ & $\begin{array}{l}\text { - Efectos tóxicos de la urea } \\
\text { Descomposición de la urea en amonio que irrita } \\
\text { la mucosa gastrointestinal }\end{array}$ \\
\hline $\begin{array}{l}\text { Estomatitis, halitosis } \\
\text { urémica y gingivitis }\end{array}$ & $\begin{array}{l}\text { - Efectos tóxicos de la urea } \\
\text { Descomposición de la urea en amonio en la } \\
\text { cavidad oral }\end{array}$ \\
\hline
\end{tabular}

Necesidad de higiene

\begin{tabular}{|c|c|}
\hline Manifestaciones clínicas & Mecanismo fisiopatológico \\
\hline - Palidez & - Anemia urémica \\
\hline - Ictericia & $\begin{array}{l}\text { - Retención de pigmentos UROCHROME que son } \\
\text { excretados a través de la piel }\end{array}$ \\
\hline $\begin{array}{l}\text { - Sequedad de piel } \\
\text { y mucosas }\end{array}$ & $\begin{array}{l}\text { - Secreción glándulas (sudor, grasa) disminuida por la } \\
\text { urea }\end{array}$ \\
\hline
\end{tabular}




\begin{tabular}{|c|c|}
\hline - Prurito & $\begin{array}{l}\text { - Piel seca } \\
\text { - Depósitos de calcio y/o fosfato en la piel } \\
\text { - Efecto de la toxicidad de la urea en la parte distal de } \\
\text { los nervios }\end{array}$ \\
\hline $\begin{array}{l}\text { - } \quad \text { Púrpura y } \\
\text { equimosis }\end{array}$ & $\begin{array}{l}\text { - Fragilidad capilar aumentada } \\
\text { - Disfunción PLATELET }\end{array}$ \\
\hline - Escarcha urémica & - Excreción de urea o de cristales de ureato por la piel \\
\hline
\end{tabular}

Necesidad de moverse

\begin{tabular}{|c|c|}
\hline Manifestaciones clínicas & \multicolumn{1}{c|}{ Mecanismo fisiopatológico } \\
\hline - Hipocalcemia & $\begin{array}{l}\text { Hiperfosfatemia secundaria a la disminución de la } \\
\text { excreción renal } \\
\text { Reabsorción gastrointestinal disminuida } \\
\text { secundario a la disminución de la conversión de la } \\
\text { vitamina D }\end{array}$ \\
\hline - Osteodistrofia & $\begin{array}{l}\text { Aumento de la secreción de la hormona } \\
\text { paratiroidea (PTH) que aumenta la movilización }\end{array}$ \\
\hline - Calcificación de los & $\begin{array}{l}\text { Cristales de fosfato cálcico se depositan en los } \\
\text { tejidos blandos y en otras estructuras }\end{array}$ \\
\hline tejidos blandos & \begin{tabular}{l} 
\\
\hline
\end{tabular}
\end{tabular}

Necesidad de evitar peligros

\begin{tabular}{|c|l|}
\hline Manifestaciones clínicas & \multicolumn{1}{c|}{ Mecanismo fisiopatológico } \\
\hline - Anemia & \begin{tabular}{l} 
- Secreción de eritropoyetina disminuida \\
- $\begin{array}{l}\text { Pérdidas sanguíneas gastrointestinales, mucosas, } \\
\text { diálisis) } \\
\text { - Supervivencia de las células disminuida y acción } \\
\text { del ácido fólico afectado por los efectos tóxicos de } \\
\text { la urea }\end{array}$ \\
\hline $\begin{array}{l}\text { Alteraciones en la } \\
\text { coagulación }\end{array}$
\end{tabular} \\
\hline
\end{tabular}




\begin{tabular}{|c|c|}
\hline - Infecciones & $\begin{array}{l}\text { - Alteración del sistema inmunológico secundario a } \\
\text { la toxicidad de la urea }\end{array}$ \\
\hline $\begin{array}{l}\text { - Gastritis, úlceras y } \\
\text { sangrado }\end{array}$ & $\begin{array}{l}\text { - Efectos tóxicos de la urea } \\
\text { - Descomposición de la urea en amonio que produce } \\
\text { pequeñas úlceras en la mucosa gastrointestinal }\end{array}$ \\
\hline $\begin{array}{l}\text { - Letargia, } \\
\text { confusión, coma e } \\
\text { irritabilidad }\end{array}$ & $\begin{array}{l}\text { - La toxicidad urémica produce una encefalopatía } \\
\text { - Arémica } \\
\text { - Acidosis metabólica }\end{array}$ \\
\hline $\begin{array}{l}\text { Temblores, } \\
\text { nerviosismo y } \\
\text { convulsiones }\end{array}$ & $\begin{array}{l}\text { - Alteraciones electrolíticas } \\
\text { - Encefalopatía urémica }\end{array}$ \\
\hline $\begin{array}{l}\text { - Neuropatía } \\
\text { periférica }\end{array}$ & $\begin{array}{l}\text { - Conducción nerviosa motora y sensitiva disminuida } \\
\text { secundaria a la toxicidad urémica }\end{array}$ \\
\hline $\begin{array}{l}\text { - Disminución del } \\
\text { habla, de la }\end{array}$ & $\begin{array}{l}\text { - Encefalopatía urémica } \\
\text { - Desequilibrio electrolítico }\end{array}$ \\
\hline $\begin{array}{l}\text { - concentración y } \\
\text { alteración de las } \\
\text { percepciones }\end{array}$ & $\begin{array}{l}\text { - Acidosis metabólica } \\
\text { - Tendencia a desarrollar edema cerebral }\end{array}$ \\
\hline
\end{tabular}




\section{Figuras:}

Figura 1. Autorregulación renal

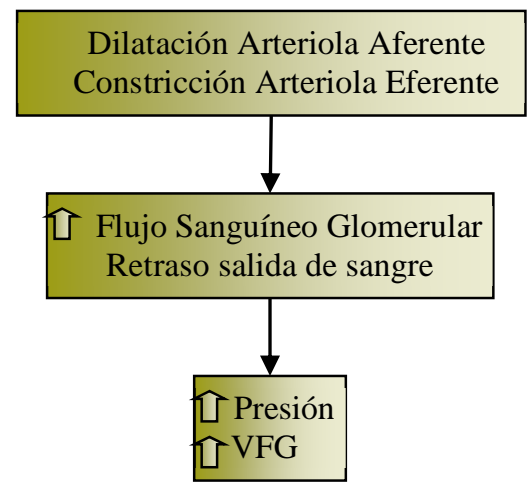

Figura2. Sistema renina-angiotensina

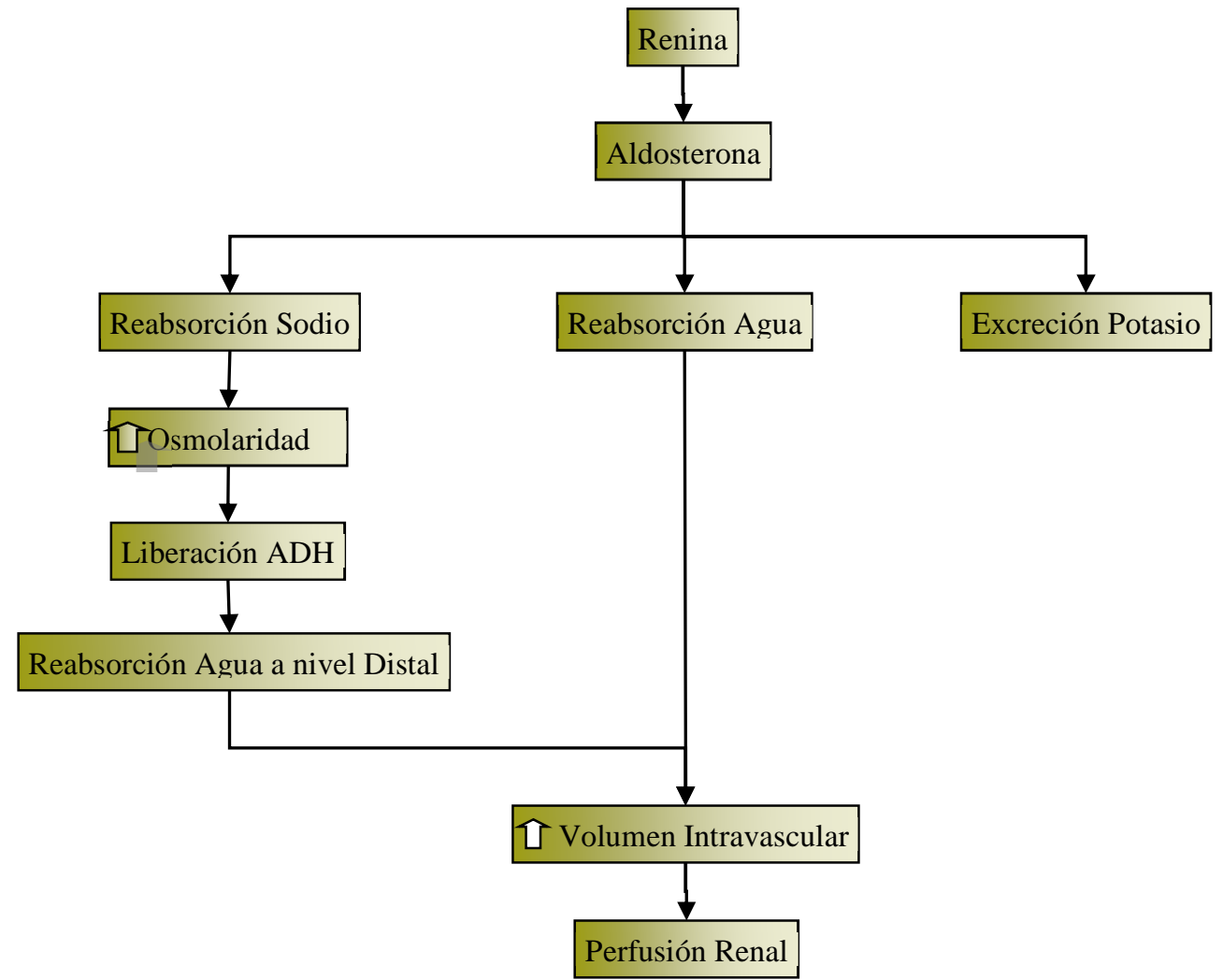


Figura 3. Escala RIFLE

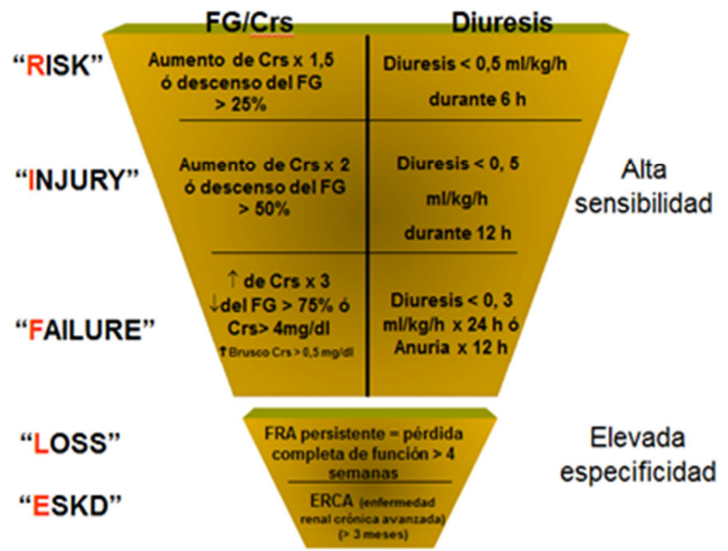

Basada en: Bellomo R, Ronco C, Kellum JA, Metha RL, Palevsky P. Acute renal failure-definition, outcome measures, animal models, fluid therapy and information technology needs: the second International Consensus Conference of the Acute Dialysis Quality Initiative (ADQI) Group ${ }^{(13)}$. 